\title{
MICROSATÉLITES: SUS APLICACIONES EN LA CONSERVACIÓN DE LA BIODIVERSIDAD
}

\author{
E. G. González*
}

\section{RESUMEN}

En los últimos años, la utilización de microsatélites como marcador molecular en estudios de conservación de la biodiversidad ha aumentado considerablemente, debido a dos características principales: su elevada tasa de polimorfismo y la posibilidad de poder trabajar con reducidas cantidades de ADN, que se pueden obtener sin que resulte necesario el sacrificio de ejemplares (es decir, no invasivas). Son particularmente útiles para resolver diversas cuestiones relacionadas con la conservación de la diversidad genómica, como la detección de posibles cuellos de botella, medida del flujo génico e hibridación entre poblaciones, determinación de paternidades, asignación de individuos a su población de origen y determinación de la estructura poblacional, entre otras. En este trabajo se revisa la información existente sobre microsatélites (características, ventajas y limitaciones), y en particular cuándo son más adecuados para resolver determinados problemas planteados para un organismo. Así mismo, se presentan consideraciones futuras de estos y otros marcadores alternativos.

Palabras clave: Microsatélites, biodiversidad, conservación, genética de poblaciones, revisión.

\begin{abstract}
Microsatellites: Application to biodiversity conservation

Microsatellites have been increasingly used as molecular marker in biodiversity conservation studies in the last years, due to two main characteristics: high level of polymorphism, and the possibility of working with low quantities of DNA which can be obtained without the sacrificing of the specie to study (non invasive samples). Microsatellite markers appear particularly useful to deal with issues such as detecting bottleneck, measuring gene flow and hybridization between populations, determining paternity, assigning individuals to their population of origin and determining population structure. This article highlights information about microsatellites (characteristics, advantages and limitations) that can be used to determine if they are suitable markers to solve particular problems about an organism. It also outlines future considerations of these and other alternative markers.
\end{abstract}

Key words: microsatellites, biodiversity, conservation genetics, genetic population, review.

1 Departamento de Biodiversidad y Biología Evolutiva, Museo Nacional de Ciencias Naturales, (CSIC), José Gutiérrez Abascal, 2. 28006, Madrid, España. e-mail: elenag@mncn.csic.es 


\section{Introducción}

La conservación de las especies con poblaciones fragmentadas y en peligro de extinción pasa por un conocimiento previo y profundo de su dinámica y estructura metapoblacional, lo cual supone la determinación de la variabilidad genética dentro y entre poblaciones. Los marcadores moleculares nos permiten, por ejemplo, identificar poblaciones con una diversidad genética reducida y generalmente más vulnerables a un posible cambio ambiental, así como distinguir subpoblaciones genéticamente diferenciadas del resto para dirigir los esfuerzos de conservación hacia ellas. Del mismo modo, permiten descubrir genealogías génicas y conocer el grado de parentesco entre individuos a fin de programar cruzamientos que prevengan niveles de consanguinidad altos que puedan llevar a la depresión biológica y su posible extinción, etc.

Sin embargo la popularidad en el uso de distintos marcadores ha variado con el tiempo. La aplicación por primera vez de las técnicas de electroforesis de enzimas (separación en un campo eléctrico de proteínas) a mediados de los años 60 (Harris, 1966; Hubby \& Lewontin, 1966) supuso un gran avance en la detección de variabilidad genética; aunque paulatinamente se han sustituido por otros marcadores moleculares que detectan polimorfismos a nivel de ADN, como los RFLPs (Restriction Fragment-Length Polymorphisms) (Sunnucks, 2000).

Sin embargo, con el desarrollo y generalización de la PCR (Polymerase Chain Reaction) (Saiki et al, 1985; Mullis et al, 1986) tomaron ventaja una serie de marcadores que de otra forma no podrían haber progresado, como es el caso de los microsatélites y minisatélites. Así pues, la publicación en 1989 de tres trabajos sobre el aislamiento y caracterización de microsatélites (Litt \& Lutty, 1989; Tautz, 1989; Weber \& May, 1989), supuso el inicio del empleo de forma cada vez más generalizada de este marcador, y que ha terminado revolucionado los campos de la biología molecular, la genética cuantitativa y la genética de poblaciones.

Los microsatélites son marcadores codominantes que consisten en secuencias cortas (de 1 a 6 bases nucleotídicas) repetidas en tándem un alto número de veces, con una tasa de variación en el número de copias entre individuos; que gracias a la técnica de PCR son fácilmente automatizables y además permite utilizar muestras no invasivas. Debido a la elevada tasa de polimorfismo que suelen presentar, aportan información muy útil que se puede aplicar para resolver problemas tanto especí- ficos como individuales (Bruford \& Wayne, 1993; Jarne \& Lagoda, 1996). Por ello se han convertido, en los últimos años, en uno de los marcadores más utilizados en estudios de conservación y manejo de especies en peligro de extinción.

En el caso de los minisatélites el tamaño de la unidad que se repite puede llegar a los 200 pares de bases (con tamaños de alelos de hasta 50 kilobases) por lo que la efectividad de la PCR queda limitada.

Otros ejemplos de marcadores moleculares son los RAPDs (Random Amplification of Polymorphic DNA) y los AFLPs (Amplified Fragment Length Polymorphism) que en combinación con la PCR tienen la ventaja de ser de fácil manejo en el laboratorio y con costes relativamente bajos; pero presentan la desventaja de ser dominates y generados al azar (difícilmente reproducibles). Recientemente un nuevo tipo de marcadores, empiezan a aparecer en escena, son los SNPs (Single Nucleotide Polymorphism) (Vignal et al., 2002) que aunque aún no existen muchos ejemplos aplicados a problemas de conservación podrían jugar un papel importante en el futuro.

En este trabajo de revisión se resumen las características de los microsatélites en los genomas de eucariotas y sus patrones de evolución. También se exponen de forma esquemática los principales métodos de obtención de microsatélites específicos para aquellas especies en las que no se ha descrito la PCR. Finalmente se presentan diversos ejemplos en los que se ha aplicado el estudio de los microsatélites a distintos aspectos de la genética de poblaciones, destacando aquellos específicamente enfocados a aspectos relacionados con estudios de conservación.

\section{Definición y características}

Los microsatélites, al igual que los minisatélites, son marcadores repetitivos o VNTRs (Variable Number of Tandem Repeats), constituidos por secuencias cortas de 1 a 6 pares de bases nucleotídicas que se repiten en tándem un elevado número de veces (SSRs, Short Sequence Repeats). Presentan herencia mendeliana simple, son codominantes (pudiéndose diferenciar los individuos homocigóticos de los heterocigóticos), y con un número muy alto de alelos en un mismo locus (altamente polimórficos). Su "genotipado" resulta relativamente fácil y automatizable, con la posibilidad de realizar amplificaciones múltiples en una misma reacción de PCR, con resultados fáciles de analizar, fiables y repetitivos. 
Son muy abundantes en los genomas nucleares de todos los eucariotas, algunos procariotas, en los genomas de cloroplastos de las plantas (McCauley, 1995; Provan et al., 2001), e incluso hay evidencias de su presencia en algunos genomas mitocondriales de vertebrados (Hoelzel et al., 1993a; Zardoya \& Meyer, 1998).

Se ha estimado que la tasa de mutación en los microsatélites varía entre $10^{-2}$ y $10^{-5}$ por generación (Weber \& Wong, 1993; Chakraborty et al., 1997), y el mecanismo que explica mejor su alto grado de polimorfismo de tamaño es la acumulación de errores producidos por el deslizamiento de la polimerasa durante la replicación del ADN (Schlötterer y Tautz, 1992; Strand et al., 1993; Tautz y Schlötterer, 1994; Kruglyak et al., 1998; Eisen, 1999; Ellegren, 2000).

Se definen por el tipo particular de motivos de secuencia o unidades de repetición (UR), y por su longitud o número de repeticiones en una posición concreta en el cromosoma (locus). Con estos criterios se vienen clasificando generalmente en tres familias (basado en Weber, 1990):

1) Microsatélites perfectos, se definen como repeticiones en un número mínimo de 9 UR sin interrupción y sin repeticiones adyacentes ( p.e. (CA)n $n>9$ ).

2) Microsatélites compuestos, se tratan de dos o más repeticiones de al menos 9 UR ininterrumpidas. Las UR pueden ser combinaciones de motivos de un número variable de pares de bases ( p.e. (CA)n -(GA)m; n>9, m>9).

3) Microsatélites interrumpidos, son repeticiones ininterrumpidas de al menos 9 UR separadas como máximo por 4 pares de bases (p.e. $(\mathrm{CA}) n$ -Nt- (CA)n -Nt -(CA)n; $\mathrm{n}>9, \mathrm{t}<4)$.

\section{Origen y evolución de los microsatélites}

La evolución y función de los microsatélites es desconocida. Existe todavía controversia sobre qué patrones siguen en los cambios de tamaño de alelos de una generación a otra, que factores determinan la tasa de mutación (Hancock, 1999; Li et al., 2002; Sibly et al., 2003), y la función que pueden desempeñar debido al hecho de que se encuentran tanto en regiones codificantes como no codificantes (predominantemente en estas últimas), (Tautz et al., 1986; Kashi et al., 1997; Kashi \& Soller, 1999; Li et al., 2002).

Los modelos que se han considerado más relevantes para explicar la evolución de los microsaté- lites son tres: el modelo de alelos infinitos (Infinite Allele Model, IAM) (Kimura \& Crow, 1964), el modelo del K-alelo (K-allele model, KAM) (Crow \& Kimura, 1970) y el modelo del salto mutacional (Stepwise Mutation Model, SMM) (Kimura \& Otha, 1978). Este último es el que se ha aplicado más ampliamente, e implica que las mutaciones suponen la pérdida o ganancia de una sola unidad de repetición, y por lo tanto, las mutaciones tienen lugar sobre alelos ya presentes en la población, es decir, que mantienen cierta "memoria" sobre el estado alélico anterior (Weber \& Wong, 1993; Goldstein et al., 1995; Balloux \& Goudet, 2002). Este modelo se definió inicialmente para explicar la distribución de las frecuencias alélicas en alozimas, pero se aplica mejor a los microsatélites, donde se asume que las mutaciones ocurren más fácilmente de una manera regular. Por el contrario, según el modelo IAM, las unidades de repetición mutan en un número variable y por lo tanto el resultado no depende del estado alélico previo de la población. Por último, el modelo KAM asume $\mathrm{k}$ posibles estados alélicos en una población y cada alelo tiene una probabilidad constante $(\mu /(\mathrm{k}-1))$ de mutar de entre los $\mathrm{k}-1$ estados alélicos restantes.

Hay evidencias de que el aumento del tamaño del alelo ocurre de forma más frecuente que su disminución, pero se desconoce si hay fuerzas selectivas en el mantenimiento y generación de los microsatélites (Primmer et al., 1996; Broede \& Ellegren, 1999).

Desde una perspectiva filogenética, se ha podido profundizar mejor en la dinámica evolutiva de los microsatélites. A pesar de que los microsatélites poseen altas tasas de mutación, las regiones flanqueantes están más conservadas, y por tanto se pueden utilizar para establecer hipótesis filogenéticas entre especies cercanas. De esta forma se han resuelto filogenias a nivel de familia, para los cíclidos (Zardoya et al., 1996) y salmónidos (Koskinen et al., 2002), de subfamilia para las avispas Polistinae (Zhu et al., 2000); y a nivel específico para el sapo corredor Bufo calamita (Rowe et al., 1998) o el cangrejo de herradura Limulus polyphemus (Ortí et al., 1997), entre otros.

Por otro lado, existe también una gran variabilidad en la cantidad de secuencias de microsatélites en los genomas de diferentes especies en términos de frecuencia, longitud y tipo de repetición (Tóth et al., 2000; Neff \& Gross, 2001). Por ejemplo, se sabe que los microsatélites son bastante abundantes en peces (DeWoody \& Avise, 2000), insectos himenópteros (Choundary et al., 1993) y mamíferos (Forbes et al., 1995). Sin embargo, son relativamente raros en los genomas de aves (Primmer et al., 
1997), en plantas (los cuales presentan cinco veces menor cantidad de microsatélites que los mamíferos) (Lagercrantz et al., 1993) y en lepidópteros (Saccheri \& Bruford, 1993).

La amplificación cruzada para un mismo locus suele mantenerse cuando se trabaja con taxones cercanos pertenecientes al mismo género o a géneros separados recientemente (Scribner \& Pearce, 2000), mientras que la información que ofrecen los microsatélites se suele perder a niveles suprafamiliares. Sin embargo, existen muchas diferencias entre distintos grupos de organismos (ver apartado ventajas e inconvenientes)

\section{Obtención, clonación y aislamiento de microsatélites}

Existen numerosos métodos disponibles para el aislamiento de microsatélites (ver Zane et al., 2002 para revisión), y la elección de uno u otro depende de las limitaciones técnicas de que se dispongan, de los objetivos que se busquen para el trabajo y de las características de la especie a estudiar (ya hemos señalado las diferencias que existen en cuanto a la cantidad de secuencias de microsatélites a lo largo de los taxones).

El método más sencillo consiste en la búsqueda e identificación de microsatélites mediante sondas en librerías genómicas o genotecas completas (Rassmann et al., 1991). Esta estrategia de aislamiento es más efectiva en grupos en los que la cantidad de secuencias de microsatélites es alta o cuando se requiere un número bajo de microsatélites, como por ejemplo en estudios de asignación de parentesco.

Brevemente, se digiere ADN de alto peso molecular con distintas enzimas de restricción y se liga a un vector previamente linearizado, para poder ser transformados en bacterias competentes. Una vez asegurada una buena representación del genoma del individuo, la búsqueda de microsatélites se realiza mediante la transferencia de las colonias a membranas de nylon y su hibridación con oligos marcados o sondas, que contienen microsatélites (Figura 1).

En el caso de que se necesite mejorar la efectividad del método, bien porque se trabaja con taxones que presentan cantidades bajas de secuencias de microsatélites, o porque se necesitan grandes cantidades de los mismos de una o varias especies, se han desarrollado métodos alternativos para disminuir el tiempo invertido y aumentar el número de microsatélites aislados.

Algunas de estas alternativas son modificaciones del método de amplificación de polimorfismos al azar debido a la abundancia de regiones repetidas que se observa en los productos amplificados por cebadores de RAPDs.

Sin embargo, el método más ampliamente utilizado es la construcción de genotecas enriquecidas (Ostrander et al., 1992; Toonen, 1997; Hamilton et al., 1999; Brown et al., 2001 y Carleton et al., 2002). La diferencia con el primer método descrito es que los fragmentos utilizados para construir la genoteca ya son ricos en microsatélites (Figura 1). En este caso el ADN digerido se une a ligadores, que sirven como lugares de unión para los cebadores en la amplificación por PCR y permiten su posterior ligación al vector. El siguiente paso consiste en la hibridación de sondas marcadas, generalmente con biotina, a regiones del ADN con microsatélites, y al mismo tiempo la unión de estas sondas a bolas magnéticas. Gracias a esto, al someter esta mezcla a un campo magnético se pueden capturar y separar fragmentos ricos en microsatélites, para su posterior clonación.

\section{Ventajas e inconvenientes de los microsatélites}

Las ventajas que ofrecen los microsatélites se deben en parte a la posibilidad de la amplificación en cadena de la polimerasa (PCR), ya que el tejido que se utiliza no necesita ser de mucha calidad, e incluso ADN en estado avanzado de degradación es suficiente para ser analizado. En este caso aventaja a otras técnicas que también utilizan ADN nuclear, como son RFLPs o los RAPDs. Los microsatélites son marcadores apropiados para el estudio de genética de poblaciones en especies en peligro de extinción porque se pueden amplificar a partir de muestras de pelo, heces, hueso (Höss \& Paabo, 1993), plumas (Ellegren, 1993), biopsias, sangre, etc., que no suponen el sacrificio de los individuos a estudiar. De esta forma es posible analizar el área de distribución, movimiento y estructura social de las poblaciones sin tener que tomar contacto directo con los individuos (Beheregaray et al., 2000; Sloane et al., 2000; Ernest, et al., 2000). Además, puede utilizarse con muestras conservadas en museos, colecciones privadas, etc., de muchos años de antigüedad. Asimismo, puesto que es posible amplificar pequeñas cantidades, se puede ampliar el campo de aplicación a especies de muy pequeño tamaño, como insectos, etc.

Por el contrario, marcadores como las aloenzimas se obtienen de músculo, hígado, riñón, etc., lo que puede suponer el sacrificio del individuo, debido a que la expresión isoenzimática normalmente 


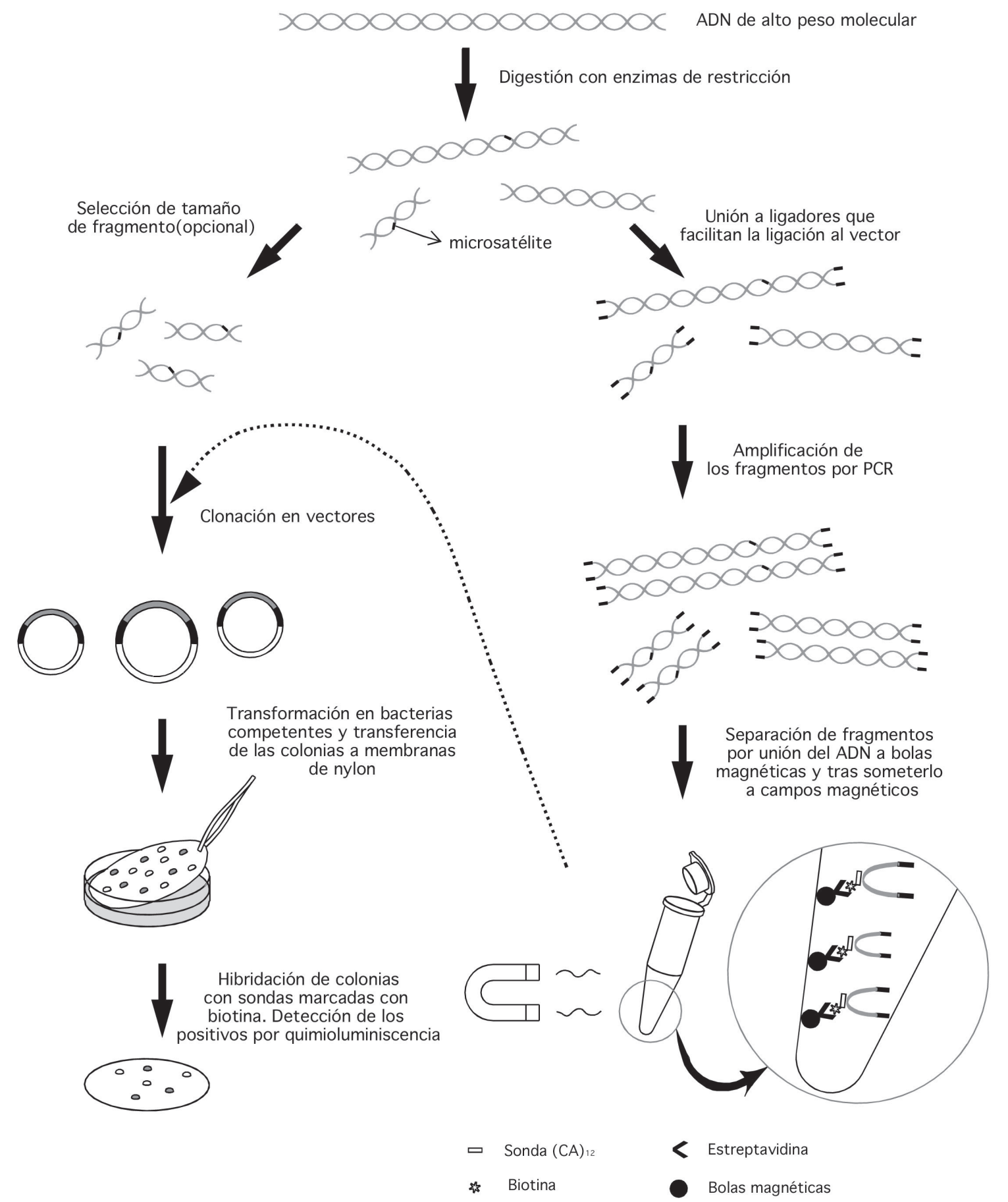

Fig. 1-. Representación esquemática del método de aislamiento de microsatélites. Izquierda: método basado en librerías genómicas completas (Rassmann et al., 1991). Derecha: método basado en librerías genómicas enriquecidas (Ostrander et al., 1992; Toonen, 1997; Hamilton et al., 1999; Brown et al., 2001; Carleton et al., 2002).

Fig. 1-. Schematic representation of the microsatellite isolation methods. Left: method based on complete libraries (Rassmann et al., 1991). Right: method based on enriched libraries (Ostrander et al., 1992; Toonen, 1997; Hamilton et al., 1999; Brown et al., 2001; Carleton et al., 2002). 
es específica de tejido. Hay casos, sin embargo, en que se han podido utilizar otros tejidos no destructivos con alozimas, como mucus de pescado, almohadillas de aves, biopsias o sangre, etc.

Además de los métodos clásicos de análisis de genética de poblaciones basados en frecuencias alélicas y obtención de distancias genéticas, hoy día se empiezan a aplicar diversos métodos para el análisis estadístico de microsatélites, como máxima verosimilitud, coalescencia y estadística Bayesiana, (Luikart \& England, 1999; Beaumont \& Bruford, 1999; Whittaker et al., 2003) y muchos programas estadísticos basados en estos métodos se pueden encontrar fácilmente en Internet (Tabla1).

En cuanto a los inconvenientes, uno de ellos es que en muchos casos resulta necesario aislar y caracterizar marcadores específicos para la especie objeto de estudio, lo que supone un elevado esfuerzo en términos de tiempo y recursos económicos. Esto puede resultar un inconveniente añadido para ciertos grupos de organismos en los cuales su aislamiento y obtención resulta muy difícil técnicamente, o bien porque los microsatélites aislados para un grupo sean inadecuados para grupos próximos (Primmer \& Merilä, 2002; Garner, 2002). Sin embargo, en algunos casos los cebadores desarrollados para una especie se pueden utilizar en taxones cercanos sin tener que aislarlos específicamente para cada especie. Hay ejemplos de mamíferos (Moore et al., 1991; Schlötterer et al., 1991), tortugas (FitzSimmons et al., 1995) aves (Primmer et al., 1996; Primmer \& Ellegren, 1998) y peces

Tabla 1.- Programas utilizados para el análisis de frecuencias alélicas o haplotipos en estudios de genética de poblaciones. No todos los programas son exclusivos para el análsis de datos de microsatélites. Como pie de tabla se indica la referencia bibliográfica para algunos programas, los cuales son de acceso libre en las direcciones de internet que se indican.

Table 1.- Computer programs used in genetic population studies for analyzing allele frequency data or haplotype data. Many of these programs are not limited to microsatellite data analysis. The footnotes show the references for some of the programs, which are freely available in the websites cited.

\begin{tabular}{|c|c|}
\hline Nombre del programa & Dirección de internet \\
\hline GENEPOP $^{1}$ & http://wbiomed.curtin.edu.au/genepop/ \\
\hline FSTAT $^{2}$ & http://www.unil.ch/izea/softwares/fstat.html \\
\hline STRUCTURE $^{3}$ & http://pritch.bsd.uchicago.edu/ \\
\hline POPULATIONS & http://www.cnrs-gif.fr/pge/bioinfo/populations/index.php?lang=fr \\
\hline GENETIX & http://www.univ-montp2.fr/\%7Egenetix/genetix/genetix.htm \\
\hline ARLEQUIN & http://lgb.unige.ch/arlequin/ \\
\hline MICROSAT & http://hpgl.stanford.edu/projects/microsat/ \\
\hline MIGRATE $^{4}$ & http://evolution.genetics.washington.edu/lamarc.html \\
\hline BOTTLENECK $^{5}$ & http://www.ensam.inra.fr/URLB/ \\
\hline MPVal $^{6}$ & http://www.pfeg.noaa.gov/tib/staff/carlos_garza/carlossoftware.html \\
\hline BATWING $^{7}$ & http://www.maths.abdn.ac.uk/ ijw/downloads/batwing/batguide/batguide.html \\
\hline SIMCOAL & http://cmpg.unibe.ch/software/simcoal/ \\
\hline GENECLASS ${ }^{8}$ & http://www.ensam.inra.fr/URLB/ \\
\hline PARTITION $^{9}$ & http://www.univ-montp2.fr/ genetix/partitionml.htm \\
\hline LEA $^{10}$ & http://www.cnrs-gif.fr/pge/bioinfo/lea/index.php?lang=en \\
\hline RSTCALC ${ }^{11}$ & http://helios.bto.ed.ac.uk/evolgen/rst/rst.html \\
\hline KINSHIP & http://gsoft.smu.edu/GSoft.html \\
\hline RELATEDNESS & http://gsoft.smu.edu/GSoft.html \\
\hline CERVUS ${ }^{12}$ & http://helios.bto.ed.ac.uk/evolgen/ \\
\hline
\end{tabular}

Raymond, M. \& Rousset, F. 1995. Journal of Heredity, 86: 248-249. Goudet, J. 1995. Journal of Heredity, 86: 485-486.

Pritchard, J. K., Stephens, M. \& Donelly, P. J. 2000. Genetics, 185: 945-959.

Beerli, P. \& Felsenstein, J. 2001. Proceedings of the National Academy of Science, 98(8): 4563-4568.

Luikart, G \& Cornuet, J.-M. 1998. Conservation Biology, 12: 288-237.

Garza, J. C. \& Williamson, E. G. 2001. Molecular Ecology, 10: 305.

Wilson, I. J. \& Balding, D. J. 1998. Genetics, 150: 499-510.
${ }^{8}$ Cornuet, J.-M., Piry, S., Luikart, G., Estoup, A. \& Solignac, M. 1999. Genetics, 153: 1989-2000.

Castric, V., Belkhir, K., Bernatchez, L. \& Bonhomme, F. 2002. Heredity, 89: 27-35.

${ }^{10}$ Langella, O., Chikhi, L. \& Beaumont, M. A. 2001. Molecular Ecology Notes, 1: $357-358$

${ }^{1}$ Goodman, S. J. 1997. Molecular Ecology, 6: 881-885.

${ }^{12}$ Marshall, T. C., Slate, J., Kruuk, L. E. B. \& Pemberton, J. M. 1998. Molecular Ecology, 7: 639-655. 
(Zardoya et al., 1996; Rico et al., 1996; DeWoody \& Avise, 2000) en los que se ha podido amplificar en especies separadas entre 100 y 400 Ma.

La utilización de los cebadores de una especie, para amplificar por PCR microsatélites en especies cercanas, tiene como principal inconveniente la aparición de alelos nulos, debido a sustituciones, delecciones e inserciones en las secuencias que corresponden a los sitios de unión de los cebadores específicos. Esto puede implicar la asignación errónea de individuos heterozigóticos como homozigóticos, y puede suponer a la larga la acumulación de grandes errores en los análisis de datos si los alelos nulos son abundantes.

Asimismo, y derivado del uso de material de baja calidad, como heces, pelo, etc., nos podemos encontrar con el problema de la creación de "artefactos" o amplificaciones inespecíficas, que pueden conducir también a errores en el genotipado de los individuos (Taberlet et al., 1996, 1997).

Por último, la elección del modelo mutacional que se debe aplicar en cada ocasión puede resultar fundamental, puesto que numerosos parámetros en genética de poblaciones (p.e. tamaño efectivo de la población, número de inmigrantes por generación, etc.) dependen del modelo utilizado, y además esta dependencia aumenta con el nivel de polimorfismo, por lo que resulta crucial para marcadores como los mini- y microsatélites (Estoup \& Cornuet, 1999). Sin embargo, aún no existe un consenso sobre que modelo es el más apropiado.

\section{Aplicaciones del análisis de microsatélites}

\section{Cuellos de Botella}

Los cuellos de botella pueden explicar en muchos casos la existencia de niveles bajos de variabilidad genética en poblaciones naturales, debido a la fragmentación del hábitat y aislamiento.

En muchas ocasiones resulta difícil cuantificar el flujo génico entre grupos de poblaciones $y$, por tanto, detectar este tipo de procesos de aislamiento efectivo. Por ello, es importante tomar como referencia muestras de poblaciones que no hayan padecido reducciones en su tamaño, o bien de individuos antes de sufrir el cuello de botella. En estos casos, los microsatélites son uno de los marcadores más apropiados.

El análisis de los efectos de los cuellos de botella con microsatélites se ha visto beneficiado a partir del desarrollo de programas y modelos de simulación capaces de inferir cambios genéticos (pérdida de heterozigosidad, pérdida de alelos, cambios en la distribución alélica y variación en las frecuencias alélicas) causados por la reducción severa del tamaño de población efectivo $(\mathrm{Ne})$, y cuellos de botella "crípticos" (Hoelzel et al., 1993b; Luikart et al., 1998, 1999; Galtier, 2000; Spencer et al., 2000; Garza \& Williamson, 2001; Wisley et al., 2002).

En el caso del estudio de las poblaciones de cernícalo de las Islas Mauricio (Falco punctatus) (Groombridge et al., 2000), la utilización de microsatélites resultó fundamental ya que permitió analizar la variabilidad genética en muestras de tejidos de ejemplares de museo, con más de 170 años de antigüedad, así como de ejemplares actuales, utilizando extracciones de ADN de plumas. De este modo, mediante el empleo de 12 microsatélites, se comprobó que la población actual presentaba cerca del $57 \%$ de la heterocigosidad y un 55\% menos de diversidad alélica respecto a la población anterior al cuello de botella. Son valores que, aunque se han recuperado, siguen siendo inferiores a los de las poblaciones continentales no amenazadas.

Los microsatélites también han sido de gran utilidad para desarrollar métodos de identificación de cuellos de botella para las poblaciones de anfibios (Beebee \& Rowe, 2001).Para este grupo en concreto, las muestras de ADN pueden utilizarse tanto a partir del corte de un dedo (método que a su vez sirve como sistema de marcaje e identificación por captura), como de un corte pequeño en la cola de adultos (urodelos) y larvas, siendo ésta una buena fuente de material de extracción sin tener que sacrificar al individuo (Jehle \& Arntzen, 2002).

\section{HIBRIDACIÓN INTRAESPECÍFICA E INTROGRESIÓN ENTRE ESPECIES}

Numerosos son los trabajos en los que se ha utilizado la información proveniente de microsatélites combinada con la información proveniente de ADN mitocondrial (ADNmt), para la detección de introgresiones e hibridaciones entre especies (Beaumont, 2001; Pritchard \& Bruford, 2001; Randi et al., 2001; Rüber et al., 2001).

Uno de los primeros fue realizado por Roy et al. (1994), en poblaciones norteamericanas de lobo gris y coyote. Estudios previos con ADNmt no encontraron evidencias de diferenciación geográfica entre poblaciones (Lehman \& Wayne, 1991) y sin embargo la utilización de microsatélites hipervariables y con tasas de mutación mayores que el ADNmt, proporcionaron resultados muy diferentes. Se utilizaron 10 microsatélites polimórficos, caracterizados en perro doméstico, para examinar cambios en las frecuencias alélicas de poblaciones de lobo gris y coyote, en zonas donde se había docu- 
mentado hibridación entre las dos especies y en zonas donde se suponía que no había ocurrido. Como resultado se encontró que la población híbrida de lobos grises y coyotes convergía en frecuencias alélicas, y que el flujo génico era unidireccional. Sólo las frecuencias alélicas de lobo gris se veían afectadas, y aproximadamente dos coyotes reproductores por generación migraban dentro de la población local de lobos.

ASIGNACIÓN DE INDIVIDUOS A POBLACIONES. ANÁLISIS DE PATERNIDAD

Los dos casos expuestos anteriormente, se refieren a la utilización de los microsatélites a nivel poblacional. En estudios en los que se requiere un análisis a nivel individual, los microsatélites son particularmente útiles, y en general los marcadores ideales, por su elevado grado de polimorfismo, facilidad de análisis y fiabilidad en la determinación de los alelos. Es el caso de la asignación de un individuo a un probable grupo de origen, lo que permite analizar estructuras intrapoblacionales (Roques et al., 1999; Rüber et al., 2001; Beheregaray \& Sunnucks, 2001; Knutsen et al., 2003) parentescos y análisis de paternidad (Taberlet et al., 1993; Marshall et al., 1998; Wilmer et al., 1999), éxito reproductivo y estructuras sociales, (Bourque et al., 1997; Herbers \& Mouser, 1998; Tóth et al., 2002; Paxton et al., 2002).

Las hormigas, como insectos sociales, constituyen un interesante grupo para examinar la estructura social mediante el análisis de parentesco entre los componentes de una colonia. Bourke et al. (1997), analizaron un total de ocho colonias de la hormiga Leptothorax acervorum, con cinco microsatélites específicos para la especie y otros dos de una especie cercana (L. spinosior). Encontraron que todas las colonias eran poligínicas, es decir, que la progenie procedía de más de una reina reproductora y analizaron el grado de poliginia entre las distintas colonias. Además, observaron que en las colonias de $L$. acervorum existía una elevada cantidad de reinas reproductoras. Esto podría deberse a que las reinas abandonaron la colonia para fundar otras, o a que murieron antes de que los machos se desarrollaran hasta su edad adulta.

\section{Conclusiones}

Los microsatélites son uno de los marcadores moleculares más utilizados en genética de la conservación. Presentan herencia mendeliana simple, son codominantes (pudiéndose diferenciar los indi- viduos homocigóticos de los heterocigóticos) y altamente polimórficos, por lo que su utilización resulta muy adecuada tanto a nivel específico como individual. Además, gracias a la aplicación del método de PCR, se pueden utilizar muestras no invasivas, lo que supone no tener que sacrificar el individuo a analizar. Por todo ello, se están aplicando de forma muy satisfactoria para deducir procesos demográficos pasados, tales como detecciones de cuellos de botella, depresiones por consanguinidad, etc., así como flujos genéticos e hibridaciones entre especies, estructuras sociales y poblacionales, entre otros.

Por otro lado, el desarrollo y mejora de programas informáticos de fácil manejo, basados en máxima verosimilitud, coalescencia y estadística Bayesiana, como métodos alternativos a la genética de poblaciones "tradicional", está permitiendo aproximaciones más exactas a los análisis de las historias demográficas de las poblaciones.

\section{CONSIDERACIONES FUTURAS}

Los microsatélites son marcadores de muy reciente utilización que presumiblemente seguirán jugando un papel importante en estudios de conservación de la biodiversidad. La necesidad de tener que caracterizar, en algunos casos, microsatélites específicos para una gran variedad de especies a partir de genotecas completas o enriquecidas, frena la aplicabilidad de este marcador, por lo que el planteamiento futuro debe ir encaminado en aumentar el número de microsatélites aislados. Además, este incremento de información sobre los mismos, plantea nuevos enfoques en el estudio de la evolución genética en diferentes taxones a partir del análisis de patrones de frecuencia y tipos de repeticiones de microsatélites.

Otra limitación al uso de los microsatélites es que aún no se ha aceptado un modelo evolutivo que explique su origen y posterior evolución, lo que supone la falta de un consenso a la hora de definir los parámetros genéticos. En este sentido, una alternativa a los microsatélites son los AFLPs, aunque aún no se han aplicado de manera tan masiva (Mueller \& Wolfenbarger, 1999; Bensh et al., 2002). La técnica necesaria es relativamente barata y genera en poco tiempo una gran cantidad de marcadores genéticos dominantes altamente polimórficos. Son fácilmente aplicables en análisis de estructura de poblaciones, evaluación de flujo genético, e hibridación entre poblaciones. Su mayor inconveniente es que debido a que es un marcador dominante no resulta muy apropiado para análisis de asignación de individuos a una población o paternidades. 


\section{AGRADECIMIENTOS}

Me gustaría agradecer a Rafael Zardoya por el tiempo que ha dedicado en ayudarme a mejorar este artículo; y a todos aquellos que me han aportado valiosos comentarios.

E. G. González es financiada a través de una beca predoctoral del Ministerio de Ciencia y Tecnología. Este trabajo ha sido parcialmente subvencionado por los proyectos REN 20000890/GLO del Ministerio de Ciencia y Tecnología, España (Investigador principal: José Templado) y REN 20011514/GLO del Ministerio de Ciencia y Tecnología, España (Investigador principal: Rafael Zardoya).

\section{Referencias}

BAlloux, F. \& Goudet, J. 2002. Statistical properties of population differentiation estimators under stepwise mutation in a finite island model. Molecular Ecology, 11: 771-783.

Beaumont, M., Barratt, E. M., Gottelli, D., Kitchener, A. C., Daniels, M. J., Pritchard, J. K. \& BRUFORD, M. W., 2001. Genetic diversity and introgression in the Scottish wildcat. Molecular Ecology, 10: 319-336.

Beaumont, M. A. \& BRUford, M. W. 1999. Microsatellite in conservation genetics. In: Goldstein, D.B \& Schlöterer, C (eds.). Microsatellites. Evolution and applications. Oxford University Press. New York: 165183.

Beebee, T. J. C. \& Rowe, G. 2001. Application of genetic bottleneck testing to the investigation of amphibian declines: a case study with natterjack toads. Conservation Biology, 15: 266-270.

Beheregaray, L. B. \& Sunnucks, P., 2001. Fine-scale structure, estuarine colonization and incipient speciation in the marine silverside fish Odontesthes argentinensis. Molecular Ecology, 10: 2849-2866.

Beheregaray, L. B., Sunnucks, P., Alpers, D. L., Banks, S. C. \& TAYLOR, A. C., 2000. A set of microsatellite loci for the hairy-nosed wombats (Lasiorhinus krefftti and L. latifons). Conservation Genetics, 1: 89-92.

Bensch, S., Åkesson, S. \& Irwin, D. E., 2002. The use of AFLP to find an informative SNP: genetic differences across a migratory divide in willow warblers. Molecular Ecology, 11: 2359-2366.

Bourke, A. F. G., Green, H. A. A. \& Bruford, M., 1997. Parentage, reproductive skew and queen turnover in a multiple-queen ant analysed with microsatellites. Proceedings of the Royal Society of London. B, 264: 277-283.

Broede, J. \& Ellegren, H., 1999. Microsatellite evolution: polarity of subtitutions within repeats and neutrality of flanking sequences. Proceedings of the Royal Society of London. B, 266: 825-833.

Brown, M. C. L., Guttman. S. \& Glenn, T. C., 2001. Development and use of microsatellite DNA loci for genetic ecotoxicological studies of the fathead minnow (Pimephales promelas). Ecotoxicology, 10: 233-238.

BRUford, M.W. \& WAYNE, R. K., 1993. Microsatellites and their application to population genetic studies. Current Opinion in Genetics and Development, 3: 939-943.

Carleton, K. L., Streelman, J. T., Lee, B.-Y., Grarnhart, N., KidD, M. \& Kocker, T. D., 2002. Rapid isolation of CA microsatellites from the Tilapia genome. Animal Genetics, 33: 140-144.

Chakraborty, R., Kimmel, M., Stivers, D. N., DAVISON, L. J. \& DEKA, R., 1997. Relative mutation rates at di-, tri-, and tetranucleotides microsatellite loci. Proceedings of the National Academy of Sciences of the USA, 94: 1041-1046.

Choundary, M., Strassman, J. E., Solís, C. R. \& Queller, D. C., 1993. Microsatellite variation in social insect. Biochemical Genetics, 31: 87-96.

Crow, J. F. \& KimurA, M., 1979. An introduction to population genetics theory. Harper \& Row Burgess Publishing Press. New York. 591 pp.

Dewoody, J. A. \& Avise, J. C., 2000. Microsatellite variation in marine, freshwater and anadromous fishes compared with other animals. Journal of Fish Biology, 56: 461-473.

EISEN, J. A., 1999. Mechanistic basis for microsatellite instability. In: Goldstein, D. B. \& Schlöterer, C (eds.). Microsatellites. Evolution and applications. Oxford University Press. New York: 34-48.

Ellegren, H., 1993. Polymerase chain reaction (PCR) analysis of microsatellites - a new approach to studies of genetic relationships in birds. Auk, 109: 886-895.

Ellegren, H., 2000. Microsatellite mutations in the germline: implications for evolutionary inference. Trends in Genetics, 16: 551-558.

Ernest, H. B., Penedo, M. C. T., May, B. P., Syvanen, M. \& Boyce, W. M., 2000. Molecular tracking of mountain lions in the Yosemite Valley region in California: genetic análisis using microsatellites and faecal DNA. Molecular Ecology, 9: 433-441.

Estoup, A. \& Cornuet, J.-M., 1999. Microsatellite evolution: inferences from population data. In: Goldstein, D. B \& Schlöterer, C (eds.). Microsatellites. Evolution and applications. Oxford University Press. New York: 49-60.

Fitzsimmons, N. N., Moritz, C. \& Moore, S. S., 1995. Conservation and dynamics of microsatellite loci over 300 million years of marine turtle evolution. Molecular Biology and Evolution, 12: 432-440.

Forbes, S. H., Hogg, J. T., Buchanan, F. C., Crawford, A. M. \& AllendoRF, F. W., 1995. Microsatellite evolution in congeneric mammals: domestic and bighorn sheep. Molecular Biology and Evolution, 12: 11061113. 
Galtier, N., Depaulis, F. \& Barton, N., 2000. Detecting bottlenecks and selective sweeps from DNA sequence polymorphism. Genetics, 155: 981-987.

GARNER, T. W. J., 2002. Genomic size and microsatellites: the effect of nuclear size on amplification potential. Genome, 45: 212-215.

Garza, J. C. \& Williamson, E. G., 2001. Detection of reduction in population size using data from microsatellite loci. Molecular Ecology, 10: 305-318.

Goldstein, D. B., Ruiz, A., Cavalli-Sforza, L. L. \& FELDMAN, M. W., 1995. An evaluation of genetic distances for use with microsatellite loci. Genetics, 139: 463-471.

Groombridge, J. J., Jonest, C. G., Bruford, M. W. \& Nichols, R. A., 2000. "Ghost" alleles of the Mauritius kestrel. Nature, 403: 616-617.

Hamilton, M. B., Pincus E. L. \& Fleisher, R. C., 1999. Universal linker and ligation procedures for construction of genomic DNA libraries enriched for microsatellites. Biotechniques, 27: 500-515.

HANCOCK, J. M., 1999. Microsatellites and other simple sequences: genomic context and mutational mechanisms. In: Goldstein, D. B \& Schlöterer, C. (eds.). Microsatellites. Evolution and applications. Oxford University Press. New York: 1-9.

Herbers, J. M. \& Mouser, R. L., 1998. Microsatellite DNA markers reveal details of social structure in forest ants. Molecular Ecology, 7: 299-306.

Hoelzel, A. R., Halley, J., O’Brien, S. J., Campagna, C., Arnbom, T., Boeuf, B., Ralls, K. \& Dover, G. A., 1993b. Elephant seal genetic variation and the use of simulation models to investigate historical population bottlenecks. Journal of Heredity, 84: 443-449.

Hoelzel, A. R., Hancock, J. M. \& Dover, G. A., 1993 a. Generation of VNTRs and heteroplasmy by sequence turnover in the mitochondrial control region of two elephant seal species. Journal of Molecular Evolution, 37: 190-197.

Höss, M. \& PAABO, S., 1993. DNA extraction from Pleistocene bones by a silica-based purification method. Nucleic Acids Research, 21: 3913-3914.

HubBy, J. L. \& Lewontin, R. C., 1966. A molecular approach to the study of genic heterozygosity in natural populations. The number of alleles at different loci in Drosophila pseudoobscura. Genetics, 54: 577-594.

JARNE, P. \& LAGODA, P. J. L., 1996. Microsatellites, from molecules to population and back. Trends in Ecology and Evolution, 11: 424-429.

JEHLE, R. \& ARNTZEN, J. W., 2002. Review: microsatellite markers in amphibian conservation genetics. Herpetological Journal, 12: 1-9.

Kashi, Y., KING, D. \& Soller, M., 1997. Simple repetitive DNA sequence repeats as a source of quantitative genetic variation. Trends in Genetics, 13: 74-78.
Kashi, Y. \& Soller, M., 1999. Functional roles of microsatellites and minisatellites. In: Goldstein, D. B \& Schlöterer, C. (eds.). Microsatellites. Evolution and applications. Oxford University Press. New York: 10-23.

KimurA, M. \& CROW, J. F., 1964. The number of alleles that can be mantained in a finite population. Genetics, 49: 725-738.

Kimura, M. \& OTHA, T., 1978. Stepwise mutation model and distribution of allelic frequencies in a finite population. Proceedings of the National Academy of Sciences of the USA, 75: 2868-2872.

Knutsen, H., Jorde, P. E., André, C. \& Stenseth, N. C., 2003. Fine-scale geographical population structuring in a highly mobile marine species: the Atlantic cod. Molecular Ecology, 12: 385-394.

Koskinen, M. T., Nilsson, J., Veselov, A. J. E., Potutkin, A. G., Ranta, E. \& Primmer, C. R., 2002. Microsatellite data resolve phylogeographic patterns in European grayling, Thymallus thymallus, Salmonidae. Heredity, 88: 391-401.

Kruglyak, S., Durrett, R. T., Schung, M. D. \& AQUADRO, C. F., 1998. Equilibrium distributions of microsatellite repeat length resulting from a balance between slippage events and point mutations. Proceedings of the National Academy of Sciences of the USA, 95: 10774-10778.

Lagercrantz, U., Ellegren, H. \& Andersson, L., 1993. The abundance of various polymorphic microsatellite motifs differs between plants and vertebrates. Nucleic Acids Research, 21: 1111-1115.

Lehman, N. \& WAYNe, R. K., 1991. Analysis of coyote mitochondrial DNA genotype frequencies: estimation of effective number of alleles. Genetics, 128: 405-416.

Li, Y.-C., Abraham, B. K., Fahima, T., Avigdor, B. \& Nevo, E., 2002. Microsatellites: genomic distribution, putative functions and mutational mechanisms: a review. Molecular Ecology, 11: 2453-2465.

LitT, M. \& LuTTY, J. A., 1989. A hypervariable microsatellite revealed by in invitro amplification of dinucleotide repeats within the cardiac muscle actine gene. American Journal of Human Genetic, 44: 397-401.

Luikart, G., Cornuet, J. M. \& Allendorf, F. W., 1999. Temporal changes in allele frequencies provide estimates of population bottleneck size. Conservation Biology, 13: 523-530.

LUIKART, G. \& ENGLAND, P. R., 1999. Statistical analysis of microsatellite DNA data. Trends in Ecology and Evolution, 14: 253-256.

Luikart, G., Sherwin, W. B., Steele, B. M. \& Allendorf, F. W., 1998. Uselfulness of molecular markers for detecting population bottlenecks via monitoring genetic change. Molecular Ecology, 7: 963-974.

Lundy, C. J., Moran, P., Rico, C., Milner, R. S. \& HewITT, G. M., 1999. Macrogeographical population 
differentiation in oceanic environments: a case study of European hake (Merluccius merluccius), a commercially important fish. Molecular Ecology, 8: 1889-1999.

Lundy, C. J., Rico, C. \& HewitT, G. M., 2000. Temporal and spatial genetic variation in spawning grounds of European hake (Merluccius merluccius) in the Bay of Biscay. Molecular Ecology, 12: 2067-2072.

Marshall, T. C., Slate, L. E., Kruuk, L. E. B. \& Pemberton, J. M., 1998. Statistical confidence for likelihood-based paternity inference in natural populations. Molecular Ecology, 7: 639-655.

McCauley, D. E., 1995. The use of chloroplast DNA polymorphism in studies of gene flow in plants. Trends of Ecology and Evolution, 10: 198-202.

Moore, S. S., Sargeant, L. L., King, T. J., Mattick, J. S., Georges, M. \& Hoetzel, D. J. S., 1991. The conservation of dinucleotide microsatellites among mammalian genomes allows the use of heterologous PCR primer pairs in closely related species. Genomics, 10:654-660.

Mueller, U. G. \& Wolfenbarger, L. R., 1999. AFLP genotyping and fingerprinting. Trends of Ecology and Evolution, 14: 389-394.

Mullis, K., Faloona, F., Scharf, S., Saiki, R., Horn, G. \& Ehrlich, H., 1986. Specific enzymatic amplification of DNA in vitro: The polymerase Chain reaction. Cold Spring Harbor Symposium Quantitative Biology, 51: 263-273.

NefF, B. D. \& Gross, M. R., 2001. Microsatellite evolution in vertebrates: inference from $\mathrm{AC}$ dinucleotide repeats. Evolution, 55: 1717-1733.

Ortí, G., Pearse, D. E. \& Avise, J. C., 1997. Phylogenetic assessment of length variation at a microsatellite locus. Proceedings of the National Academy of Sciences of the USA, 94: 10745-10749.

Ostrander, E. A., Jong, P. M., Rine, J. \& Duyk, G., 1992. Construction of small-insert genomic DNA libraries highly enriched for microsatellite repeat sequences. Proceedings of the National Academy of Sciences of the USA, 89: 3419-3423.

Paxton, R. J., Ayasse, M., Field, J. \& Soro, A., 2002. Complex sociogenetic organization and reproductive skew in a primitively eusocial sweat bee, lasioglossum malachurum, as revealed by microsatellites. Molecular Ecology, 11: 2405-2416.

Primmer, C. R. \& Ellegren, H., 1998. Patterns of molecular evolution in avian microsatellites. Molecular Biology and Evolution, 15: 997-1008.

Primmer, C. R. \& MERILÄ, J., 2002. Alow rate of crossspecies microsatellite amplification success in ranid frogs. Conservation Genetics, 3: 445-449.

Primmer, C. R., Moller, A. P. \& Ellegren, H., 1996. A wide-range survey of cross-species microsatellite amplification in birds. Molecular Ecology, 5: 365-378.
Primmer, C. R., Raudsepp, T., Chowdhary, B. P., Møller, A. P. \& Ellegren, H., 1997. A low frequency of microsatellite in the avian genome. Genome Research, 7: 471-482.

Pritchard, J. K. \& Bruford, M. W., 2001. Genetic diversity and introgression in the Scottish wildcat. Molecular Ecology, 10: 319-336.

Provan, J., Powell, W. \& Hollingsworth, P. M., 2001. Chloroplast microsatellites: new tools for studies in plant ecology and evolution. Trends in Ecology \& Evolution, 16: 142-147.

Randi, E., Pierpaoli, M., Beaumont, M., Ragni, B. \& SFORZI, A., 2001. Genetic identification of wild and domestic cats (Felis silvestris) and their hybrids using bayesian clustering methods. Molecular Biology and Evolution, 18: 1679-1693.

Rassmann, K., Schlöterer, C. \& TAutZ, D., 1991. Isolation of simple sequence loci for use in polymerasa chain reaction-based DNA fingerprinting. Electrophoresis, 12: 113-118.

Rico, C., Rico, I. \& HewitT, G., 1996. 470 millions years of conservation of microsatellite loci among fish species. Proceedings of the Royal Society of London. B, 263: 549-557.

Roques, S., Duchesne, P. \& Bernatchez, L., 1999. Potential of microsatellites for individual assignment: the North Atlantic redfish (genus Sebastes) species complex as a case study. Molecular Ecology, 8: 1703-1717.

Rowe, G., Beebee, T. J. C. \& Burke, T., 1998. Phylogeography of the natterjack toad Bufo calamita in Britain: genetic differentiation of native and translocated populations. Molecular Ecology, 7: 751-760.

Roy, M. S., Geffen, E., Smith, D., Ostrander, O. \& WAYNE, R. K., 1994. Patterns of differenciation and hybridization in North American wolflike canids, revealed by analysis of microsatellite loci. Molecular Biology and Evolution, 11: 553-570.

Rüber, L., Meyer, A., Sturmbauer, C. \& Verheyen, E., 2001. Population structure in two sympatric species of the Lake Tanganyika cichlid tribe Eretmodini: evidence for introgression. Molecular Ecology, 10: 1207-1225.

SACChERI, I. J. \& Bruford, M. W., 1993. DNA fingerprinting in a butterfly Bicyclus anynana (Satyridae). Journal of Heredity, 84:195-200.

Saiki, R. K., Scharf, S., Faloona, F., Mullis, K. B., Horn, G. T., Erlich, H. A. \& Arnheim, N., 1985 Enzymatic amplification of b-globin genomic sequences and restriction site analysis for diagnosis of sickle cell anemia. Science, 230: 1350-1354.

Schlöterer, C., Amos, B. \& Tautz, D., 1991. Conservation of polymorphic simple sequences in cetacean species. Nature, 354: 63-65.

SCHLÖTERER, C. \& TAUTZ, D., 1992. Slippage synthesis of microsatellites. Nucleic Acids Research, 20: 211-215. 
Scribner, K. T. \& Pearce, J. M., 2000. Microsatellites: evolutionary and methodological background and empirical applications at individual, population and phylogenetic levels. In: A. Barker (ed.). Molecular Methods in Ecology. Blackwell Science Limited. London: 235-271.

Sibly, R. M., Meade, A., Boxal, N., Wilkinson, M., Corne, D. W. \& WhitTaker, J. C., 2003. The structure of interrupted human AC microsatellites. Molecular Biology and Evolution, 20: 453-459.

Sloane, M. A., Sunnucks, P., Alpers, D. L., Beheregaray, L. B. \& Taylor, A. C., 2000. Highly reliable genetic identification of individual northern hairy-nosed wombats from single remotely collected hairs: a feasible censusing method. Molecular Ecology, 9: 1233-1240.

Spencer, C. C., Neigel, J. E. \& Leberg, P. L., 2000. Experimental evaluation of the usefulness of microsatellite DNA for detecting demographic bottlenecks. Molecular Ecology, 9: 1517-1528.

Strand, M., Prolla, T. A., Liskay, R. M. \& Petes, T. D., 1993. Destabilization of tracts of simple repetitive DNA in yeast by mutation affecting DNA mismatch repair. Nature, 365: 274-276.

SunNUCKS, P., 2000. Efficient genetic markers for population biology. Trends in Ecology and Evolution, 15: 199-203.

Taberlet, P., Camarra, J. J., Griffin, S., Uhres, E., Hanotte, O. \& Waits, L. P., 1997. Noninvasive genetic tracking of the endangered Pyrenean brown bear population. Molecular Ecology, 6: 869-876.

Taberlet, P., Griffin, S., Goosens, B., Questiau, S., Manceau, V., Escaravage, N. \& Waits, L. P., 1996. Reliable genotyping of samples with very low DNA quantities using PCR. Nucleic Acids Research, 24: 3189-3194.

Taberlet, P., Mattock, H., Dubois-Paganon, C. \& Bouvet, J., 1993. Sexing free-ranging brown bears Ursus arctos using hairs found in the field. Molecular Ecology, 2: 399-403.

TAUTZ, D., 1989. Hypervariability of simple sequences as a general source for polymorphic DNA markers. Nucleic Acids Research, 17: 6463-6471.

TAutz, D. \& Schlötterer, C., 1994. Simple sequences. Current Opinion Genetics and Development, 4: 832837.

Tautz, D., Trick, M. \& Dover, G., 1986. Cryptic simplicity in DNA is a major source of genetic variation. Nature, 322: 652-656.

Toonen, R., 1997. Microsatellites for Ecologists: Nonradioactive isolations and amplification protocols for microsatellite markers. Disponible en: http://biogeek.ucdavis.edu/Msats/.
Tóth, E., Strassmann, J. E., Nogueira-Neto, P., ImPeratriz-Fonseca, V. L. \& Queller, D., 2002. Male production in stingless bees: variable outcomes of queen - worker conflict. Molecular Ecology, 11: 2661-2667.

Tóth, G., GÁspari, Z. \& Jerzy, J., 2000. Microsatellites in different eukariotic genomes: survey and analysis. Genome Research, 10: 967-981.

Vignal, A., Milan, D., Sancristobal, M. \& Eggen, A., 2002. A review on SNP and other types of molecular markers and their use in animal genetics. Genetics Selection Evolution, 34: 275-305.

WeBER, J. L., 1990. Informativeness of human (dCdA)n·(dG-dT)n polymorphisms. Genomics, 7: 524530 .

Weber, J. L. \& MAY, P. E., 1989. Abundant class of human DNA polymorphisms which can be typed using polymerase chain reaction. American Journal of Human Genetic, 44: 388-396.

Weber, J. L. \& Wong, C., 1993. Mutation in human short tandem repeat polymorphisms. Human Molecular Genetic, 2: 1123-1128.

Whittaker, J. C., Harbord, R. M., Boxall, N., Mackay, I., Dawson, G. \& Sibly, R. M., 2003. Likelihood-based estimation of microsatellite mutation rates. Genetics, 164: 781-787.

Wilmer, J. W., Allen, P. J., Pomeroy, P. P., Twiss, S. D. \& Amos, W., 1999. Where have all the fathers gone? An extensive microsatellite analysis of paternity in the grey seal (Halichoerus grypus). Molecular Ecology, 8: 1417-1430.

Wisley, S. M., Buskirk, S. W., Fleming, M. A., Mcdonald, D. B. \& Ostrander, E. A., 2002. Genetic diversity and fitness in black-footed ferrets before and during a bottleneck. The Journal of Heredity, 93: 231-237.

Zane, L., Bargelloni, L. \& Patarnello, T., 2002. Strategies for microsatellite isolation: a review. Molecular Ecology, 11: 1-16.

ZardoyA, R. \& MeYer, A., 1998. Cloning and characterization of a microsatellite in the mitochondrial control region of the African side-necked turtle, Pelomedusa subrufa. Gene, 216: 149-153.

Zardoya, R., Vollmer, D. M., Craddock, C., Streelman, J. T., Karl, S. \& Meyer, A., 1996. Evolutionary conservation of microsatellite flanking regions and their use in resolving phylogeny of cichlid fishes (Pisces: Perciformes). Proceedings of the Royal Society of London. B, 263: 1589-1598.

Zhu, Y., Queller, D. C. \& Strassmann, J. E., 2000. A phylogenetic perspective on sequence evolution in microsatellite loci. Journal of Molecular Evolution, 50: 324-338. 